\title{
CENTRALIZERS OF THE FOURIER ALGEBRA OF AN AMENABLE GROUP
}

\author{
P. F. RENAUD
}

\begin{abstract}
Let $G$ be a locally compact group with Fourier algebra $A(G)$. We prove that if $G$ is amenable then every centralizer of $A(G)$ is determined by multiplication with an element of the Fourier-Stieltjes algebra of $G$. This result is then used to show that isometric centralizers correspond to characters of $G$.
\end{abstract}

1. Introduction. Let $A$ be a Banach algebra. A centralizer of $A$ is a bounded linear operator $T$ on $A$ which commutes with all the operations of left multiplication, i.e.

$$
T(x y)=x T y \text { for all } x, y \in A .
$$

If $G$ is a locally compact group, denote by $L_{1}(G)$ the group algebra of $G$ and by $M(G)$ the algebra of bounded Radon measures. In [6] Wendel proved that every centralizer of $L_{1}(G)$ is a convolution with an element of $M(G)$, i.e. there exists a unique measure $\mu \in M(G)$ such that $T x=x * \mu$ for all $x \in L_{1}(G)$.

Suppose for the moment that $G$ is Abelian with dual group $\hat{G}$. The set of functions on $G$ which are the Fourier transforms of functions in $L_{1}(\hat{G})$ is the Fourier algebra $A(G)$. Similarly by considering the transforms of elements in $M(\hat{G})$ we obtain the Fourier-Stieltjes algebra $B(G)$ (see e.g. [5, Chapter 1]). Wendel's result now shows that every centralizer of $A(G)$ is given by multiplication with an element of $B(G)$. The purpose of this note is to extend this result to noncommutative groups, the point being that although the dual group no longer exists, the algebras $A(G)$ and $B(G)$ may still be defined.

2. Definitions and notation. Throughout we shall employ the notation of [2]. Let $G$ be a (not necessarily commutative) locally compact group with identity element $e$ and let $L_{1}(G)$ denote the group algebra of $G$. The completion of $L_{1}(G)$ in the minimal regular norm is the group $C^{*}$-algebra, $C^{*}(G)$. Let $B(G)$ denote the space of all finite linear combinations of continuous positive definite functions on $G$. Regarded as the dual of $C^{*}(G)$,

Received by the editors June $28,1971$.

AMS 1970 subject classifications. Primary 43A22, 43A30; Secondary 22D15, 22D35.

Key words and phrases. Locally compact group, amenable group, Fourier algebra, centralizer.

(c) American Mathematical Society 1972 
$B(G)$ is a Banach space and even a commutative Banach algebra under pointwise multiplication. Denote by $A(G)$ the closed subspace generated by elements of $B(G)$ with compact support. It is easy to show that $A(G)$ is an ideal in $B(G)$.

For $u \in B(G)$ and $g \in G$, define ${ }_{g} u$ by ${ }_{g} u(h)=u(g h)$. Then ${ }_{g} u \in B(G)$ and $\left\|_{g} u\right\|=\|u\|$. Further if $u \in A(G)$, then so does ${ }_{g} u$.

The algebra $A(G)$ behaves in many ways like a group algebra. In general, however, it lacks one important property, the existence of an approximate identity. In [4] Leptin has shown that an approximate identity exists iff $G$ is amenable (see [3] for various alternative characterizations of amenability). We shall use this result in what follows.

\section{Centralizers of $A(G)$.}

THEOREM 1. Let $G$ be amenable and let $T$ be a centralizer of $A(G)$. Then there exists a unique element $w \in B(G)$ such that $T=L_{w}$ (where $L_{w}$ is defined by $L_{w} u=u w$ for $\left.u \in A(G)\right)$. Furthermore $\|T\|=\|w\|$.

Proof. Let $\left\{e_{\alpha}\right\}$ be an approximate identity for $A(G)$ with $\left\|e_{\alpha}\right\| \equiv 1$. The elements $T e_{\alpha}$ may be regarded as linear functionals on $C^{*}(G)$ and by continuity of $T$, the family $\left\{T e_{\alpha}\right\}$ has a $w^{*}$-accumulation point $w \in B(G)$.

Fix $u \in A(G)$. A straightforward density argument shows that $u w$ is a $w^{*}$-accumulation point of $\left\{u T e_{\alpha}\right\}$. But then by continuity of $T$,

$$
T u=\lim _{\alpha} T\left(u e_{\alpha}\right)=\lim _{\alpha} u T e_{\alpha} .
$$

This shows that $T u=u w$, i.e., $T=L_{w}$.

Since for any $g \in G$ there exists some $u \in A(G)$ with $u(g) \neq 0$, it follows that $w$ is unique. That $\|T\|=\|w\|$ follows from the existence of an approximate identity. Alternately it may be obtained at once from a result of Derighetti [1, Theorem 9].

COROLLARY 1. In the strong operator topology on $A(G), B(G)$ is a closed subset of the algebra of all bounded operators.

Proof. For if $\left\{w_{\alpha}\right\}$ is a directed set in $B(G)$ which converges strongly to an operator $S$ on $A(G)$ then for $u, v \in A(G)$,

$$
S(u v)=\lim _{\alpha} w_{\alpha} u v=(S u) v
$$

so that $S$ is a centralizer. Now apply Theorem 1 .

4. Isometric centralizers. We consider now the case when a centralizer $T$ is an isometry i.e. $\|T u\|=\|u\|$ for all $u \in A(G)$. We prove

Theorem 2. Let $G$ be amenable and let $T$ be a centralizer of $A(G)$. Then 
$T$ is an isometry iff $T=\lambda L_{\kappa}$ where $\lambda$ is some scalar of unit modulus and $\kappa$ is a character of $G$.

REMARKS. 1. It is straightforward to show that an operator $\lambda L_{\kappa}$ is an isometry so that it suffices to prove the "only if" condition.

2. For some groups $G, A(G)$ may not possess any isometric centralizers apart from those of the form $\lambda I$ since there exist amenable groups which have no nontrivial finite dimensional representations.

By Theorem 1, $T$ is of the form $L_{w}$ for some $w \in B(G)$. The proof of Theorem 2 depends on the following lemmas.

LEMMA 1. For all $g \in G, w(g) \neq 0$.

Proof. Firstly note that if $w$ defines an isometry then so does ${ }_{g} w$ for any $g$. For if $u \in A(G)$ then

$$
\left\|_{g} u u\right\|=\left\|_{g}\left(w_{g^{-1}} u\right)\right\|=\left\|w_{g^{-1}} u\right\|=\left\|_{g^{-1}} u\right\|=\|u\| .
$$

Hence it suffices to assume that $w(e)=0$ and to deduce a contradiction.

Choose $u \in A(G)$ with $u$ positive definite and $\|u\|=1$. Then $w u \in A(G)$ and $w u(e)=0$. Choose $\epsilon$ such that $0<\epsilon<1$. By [2, Lemma 4.13], we may choose $v \in A(G)$ with $v$ positive definite, $\|v\|=1$ and $\left\|v w^{\prime} u\right\|<\epsilon$. But then $\epsilon>$ $\|v v u\|=\|v u\|=\|v\|\|u\|=1$ which is a contradiction.

Lemma 2. $L_{w}$ maps $A(G)$ onto $A(G)$.

Proof. Let $N$ be the range of $L_{w}$. Since $L_{w}$ is an isometry, $N$ is a closed ideal in $A(G)$. By a Tauberian theorem [2, Corollary 3.38], it suffices to show that for all $g \in G$ there exists some $v \in N$ with $v(g) \neq 0$. Choose $u \in A(G)$ with $u(g) \neq 0$ and let $v=u w$. The result now follows from Lemma 1 .

Proof of THEOREM 2. From Lemma 2, $L_{w}^{-1}$ exists as a bounded operator on $A(G)$. If $u, v \in A(G)$ with $u=u_{1} w$ then $L_{w}^{-1}(u v)=L_{w}^{-1}\left(L_{w}\left(u_{1} v\right)\right)=$ $u_{1} v=\left(L_{w}^{-1} u\right) v$ so that $L_{w}^{-1}$ is a centralizer. From this it follows that $w^{-1} \in B(G)$ with $\left\|w^{-1}\right\|=1$.

Now $\|w\|_{\infty}=\sup _{g_{\epsilon} G}|w(g)| \leqq\|w\|=1$ and similarly $\left\|w^{-1}\right\|_{\infty} \leqq 1$. Hence $|w(g)|=1$ for all $g \in G$.

We can write $w(g)=\left(U_{g} \xi, \eta\right)$ where $g \rightarrow U_{g}$ is a unitary representation of $G$ on some Hilbert space $H$ with cyclic vector $\xi$ and with $\|w\|=\|\xi\|\|\eta\|$. For all $g \in G$,

$$
1=|w(g)|=\left|\left(U_{g} \xi, \eta\right)\right| \leqq\|\xi\|\|\eta\|=1
$$

so that by the Cauchy-Schwarz inequality, $H$ is one dimensional and there exist scalars $\lambda_{g}$ of unit modulus such that $U_{g} \xi=\lambda_{g} \eta$. Let $\lambda=\lambda_{e}^{-1}$. Then if $\kappa(g)=\lambda^{-1} \lambda_{g}$, we have that $\kappa$ is a character of $G$ and $w=\lambda \kappa$. This proves the theorem. 


\section{REFERENCES}

1. A. Derighetti, Some results on the Fourier-Stieltjes algebra of a locally compact group, Comment. Math. Helv. 45 (1970), 219-228.

2. P. Eymard, L'algebre de Fourier d'un groupe localement compact, Bull. Soc. Math. France 92 (1964), 181-236. MR 37 \#4208.

3. F. P. Greenleaf, Invariant means on topological groups and their applications, Van Nostrand Math. Studies, no. 16, Van Nostrand, Princeton, N.J., 1969. MR 40 \#4776.

4. H. Leptin, Sur l'algèbre de Fourier d'un groupe localement compact, C. R. Acad. Sci. Paris Sér. A-B 266 (1968), A1180-A1182. MR 39 \#362.

5. W. Rudin, Fourier analysis on groups, Interscience Tracts in Pure and Appl. Math., no. 12, Interscience, New York, 1962. MR 27 \#2808.

6. J. G. Wendel, Left centalizers and isomorphisms of group algebras, Pacific J. Math. 2 (1952), 251-261. MR 14, 246.

Department of Mathematics, University of Canterbury, Christchurch, New ZEALAND 